\title{
Constructing robust all-inorganic contacts enable stable perovskite solar cells with efficiencies over $20 \%$
}

\author{
Zhongwei Wu and Baoquan Sun ${ }^{*}$
}

Developing photovoltaic (PV) cells for harvesting renewable and clean solar energy is a promising strategy to meet the growing energy demand. Perovskite solar cells (PSCs), as a new player in the photovoltaic field, exhibit rapid development with an original power conversion efficiency (PCE) of $3.81 \%$ in 2009, a promising PCE of over $20 \%$ in 2014 and a record PCE of $22.1 \%$ in 2017 $[1,2]$. The explosive developments and achievements in photovoltaic effect of organometal halide perovskite shed light on the exploitation of low-cost and highly efficient photovoltaic technique. Staged PCEs over 20\% have been frequently obtained with the innovations in tailoring of perovskite absorber formation, composition and defect, charge transporting layers as well as interfacial engineering [2-4]. Currently, PCE is not a critical factor that limits its real commercial application. In addition to the potential PCE loss in up-scaling fabrication, the longterm stability is the main obstacle of pushing such kind of PSCs from laboratory to market.

For the state-of-the-art PSCs, the PCE drop may originate from the degradation of different components in a device, such as perovskite itself or the charge transporting layers. Due to the low formation energy of perovskites, they are highly susceptible to degradation in ambient. Therefore, it is essential to prohibit the perovskite photoactive layer from exposing to such environments. Such drawback can be overcome via functionalization of perovskite and rigorous encapsulation [5,6]. Additionally, the most efficient PSCs (>20\%) so far are mainly fabricated by using p-type organic semiconductors as hole transporting layers (HTLs), such as 2,2',7,7'-tetrakis-( $N$, $N$-di-p-methoxyphenylamine)-9,9'-spiro-bifluorene (spiro-OMeTAD) and polytriarylamine (PTAA). However, their relatively high cost and poor stability? compared to the n-type inorganic semiconductors $\left(\mathrm{TiO}_{2}\right.$,
$\mathrm{ZnO}$ ) are undesirable for the large-scale commercialization of PSCs. Further, these devices based on organic HTLs only can reach their peak performance in the presence of organic lithium salt and 4-tert-butylpyridine. These additives can cross into the perovskite layer and accelerate device degradation.

Constructing low-cost and robust all-inorganic contacts is a promising strategy to eliminate these unstable organic HTLs in nature. Pioneer work was conducted by Prashant and co-workers [7], employing $\mathrm{TiO}_{2}$ and $\mathrm{CuI}$ as n-type and p-type contacts, respectively. Subsequently, several all-inorganic contacts have been demonstrated including $\mathrm{TiO}_{2}-\mathrm{CuSCN}, \quad \mathrm{NiO}_{x}-\mathrm{ZnO} \quad\left(\mathrm{SnO}_{2}\right), \quad \mathrm{Li}_{0.05} \mathrm{Mg}_{0.15} \mathrm{Ni}_{0.8} \mathrm{O}-$ $\mathrm{Ti}(\mathrm{Nb}) \mathrm{O}_{x}$ and $\mathrm{TiO}_{2}-\mathrm{CuGaO}_{2}$ [8-11]. Although these allinorganic contacts yield obvious improvements in longterm stability of PSCs, proving the validity of this concept, the stabilized PCE is still away from the top class $(>20 \%)$.

Recently, Grätzel and co-workers pushed the concept of all-inorganic contacts to target stabilized PCE exceeding $20 \%$ with $\mathrm{TiO}_{2}$ and $\mathrm{CuSCN}$ as the electron and hole transporting layers (Fig. 1a) [12]. In this work, a fast solvent removal method enables the creation of compact, highly conformal CuSCN layers that facilitate efficient carrier extraction and collection. Instead of traditional spin-coating in previous researches, CuSCN solution of diethyl sulfide (DES) is drop-cast quickly while the substrate is spinning at high speed. Such dynamic deposition approach preserves the quality of perovskite layer, minimizing the damage induced by DES, which is a main barrier in CuSCN-based PSCs. CuSCN displays a higher hole mobility than commonly used spiro-OMeTAD by about three orders of magnitude, which enables it as an effective HTL even in its doping-free state.

Although successful establishment of $\mathrm{TiO}_{2}-\mathrm{CuSCN}$ all-

Institute of Functional Nano \& Soft Materials (FUNSOM), Soochow University, Suzhou 215123, China

*Email: bqsun@suda.edu.cn 

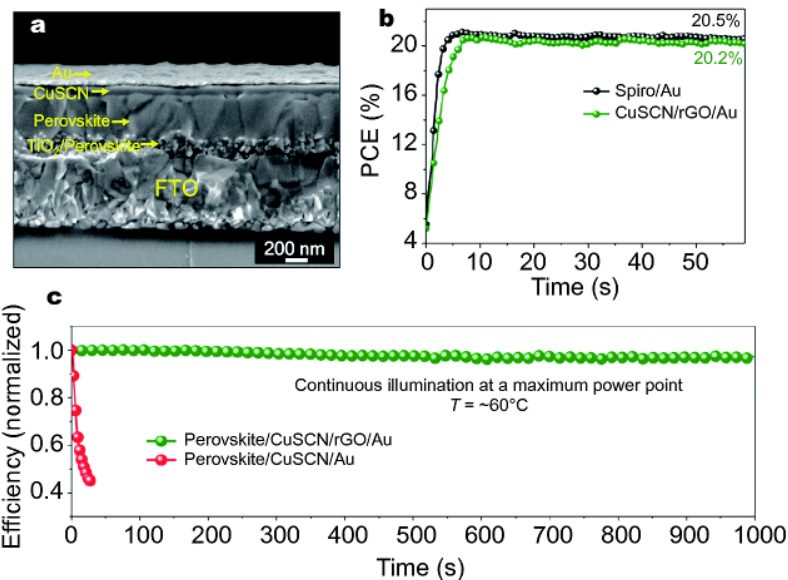

Figure 1 (a) Cross-sectional scanning electron microscopy (SEM) image of the device with all-inorganic contacts. (b) Maximum power point tracking for $60 \mathrm{~s}$, yielding stabilized PCEs of $20.5 \%$ and $20.2 \%$, respectively, for spiro-OMeTAD-based and CuSCN-based devices. (c) Operational stability of an unencapsulated CuSCN-based device with and without a thin layer of rGO (as a spacer layer between $\mathrm{CuSCN}$ and gold layers), examined at a maximum power point under continuous full-Sun illumination at $60^{\circ} \mathrm{C}$ in a nitrogen atmosphere. Reprinted with permission from Ref. [12], Copyright 2017, the American Association for the Advancement of Science.

inorganic contacts enables outstanding PCE (over 20\%) and high thermal stability at $85^{\circ} \mathrm{C}$ in ambient condition in the dark, the CuSCN/Au based devices show poor photostability, losing $>50 \%$ of their initial PCEs within $24 \mathrm{~h}$ due to an electrical potential-induced reaction of gold with the thiocyanate anions forming an undesired barrier. Graphene oxide (GO) has been firstly demonstrated by our group as an efficient hole extraction layer for PSCs [13]. The perovskite film deposited on GO exhibits high crystallization with preferred in-plane orientation of the (110) plane, which warrants its high PCE. Here, this unexpected poor operational stability is eliminated by incorporating reduced graphene oxide $(\mathrm{rGO})$ as a spacer layer (Fig. 1c), simultaneously maintaining its excellent PCE (over 20\%, Fig. 1b).

PSCs undoubtedly represent the most promising efficient and low-cost photovoltaic technique with bright future. Currently, each step of stability improvement will push it close to the real market. This study marks an important breakthrough in the concept strategy of allinorganic contacts enabling extremely stable device with shiny performance, and also highlights the significance of constructing robust contact interface with metal electrode. The impressive thermal and operational stabilities in this work will inspire future effort in achieving largescale, cost-effective and stable photovoltaic application.

Received 17 November 2017; accepted 21 November 2017; published online 20 December 2017

1 Kojima A, Teshima K, Shirai Y, et al. Organometal halide perovskites as visible-light sensitizers for photovoltaic cells. J Am Chem Soc, 2009, 131: 6050-6051

2 Yang WS, Park BW, Jung EH, et al. Iodide management in formamidinium-lead-halide-based perovskite layers for efficient solar cells. Science, 2017, 356: 1376-1379

3 Shin SS, Yeom EJ, Yang WS, et al. Colloidally prepared La-doped $\mathrm{BaSnO}_{3}$ electrodes for efficient, photostable perovskite solar cells. Science, 2017, 356: 167-171

4 Tan H, Jain A, Voznyy O, et al. Efficient and stable solutionprocessed planar perovskite solar cells via contact passivation. Science, 2017, 355: 722-726

5 Yang S, Wang Y, Liu P, et al. Functionalization of perovskite thin films with moisture-tolerant molecules. Nat Energy, 2016, 1: 15016

6 Bella F, Griffini G, Correa-Baena JP, et al. Improving efficiency and stability of perovskite solar cells with photocurable fluoropolymers. Science, 2016, 354: 203-206

7 Christians JA, Fung RCM, Kamat PV. An inorganic hole conductor for organo-lead halide perovskite solar cells. improved hole conductivity with copper iodide. J Am Chem Soc, 2014, 136: 758764

8 Qin P, Tanaka S, Ito S, et al. Inorganic hole conductor-based lead halide perovskite solar cells with $12.4 \%$ conversion efficiency. Nat Commun, 2014, 5: 3834

9 You J, Meng L, Song TB, et al. Improved air stability of perovskite solar cells via solution-processed metal oxide transport layers. Nat Nanotechnol, 2015, 11: 75-81

10 Chen $\mathrm{W}, \mathrm{Wu} \mathrm{Y}$, Yue Y, et al. Efficient and stable large-area perovskite solar cells with inorganic charge extraction layers. Science, 2015, 350: 944-948

11 Zhang $\mathrm{H}$, Wang $\mathrm{H}$, Chen $\mathrm{W}$, et al. $\mathrm{CuGaO}_{2}$ : a promising inorganic hole-transporting material for highly efficient and stable perovskite solar cells. Adv Mater, 2017, 29: 1604984

12 Arora N, Dar MI, Hinderhofer A, et al. Perovskite solar cells with CuSCN hole extraction layers yield stabilized efficiencies greater than 20\%. Science, 2017, 358: 768-771

$13 \mathrm{Wu} \mathrm{Z}$, Bai S, Xiang J, et al. Efficient planar heterojunction perovskite solar cells employing graphene oxide as hole conductor. Nanoscale, 2014, 6: 10505-10510 